\title{
Policy Implications for the COVID-19 Pandemic in Light of Most Patients ( $\geqslant 72 \%)$ Spending at Most One Night at the Hospital After Elective, Major Therapeutic Procedures
}

\author{
Richard H. Epstein ${ }^{1}$, Franklin Dexter ${ }^{2}$, Todd J. Smaka ${ }^{3}$, Keith A. Candiotti ${ }^{1}$ \\ 1. Anesthesiology, University of Miami Miller School of Medicine, Miami, USA 2. Anesthesiology, University of Iowa, \\ Iowa City, USA 3. Anesthesiology, Univeristy of Miami Miller School of Medicine, Miami, USA
}

Corresponding author: Richard H. Epstein, repstein@med.miami.edu

Received 07/17/2020

Review began 07/18/2020

Review ended 08/03/2020

Published 08/14/2020

๑) Copyright 2020

Epstein et al. This is an open access article distributed under the terms of the Creative Commons Attribution License CC-BY 4.0., which permits unrestricted use, distribution, and reproduction in any medium, provided the original author and source are credited.

\begin{abstract}
A large number of inpatients with Coronavirus disease 2019 (COVID-19) in some regions of the United States may interfere with the ability of hospitals to take care of patients requiring treatment for other conditions. Nonetheless, many patients need surgery to improve their quality of life and to prevent deterioration in health. Curtailment of services also negatively affects the financial health of hospitals and health systems. Broad policies to prohibit all "elective" surgical procedures to ensure that there is sufficient hospital capacity for pandemic patients may be unnecessarily restrictive because, for many such procedures, patients are rarely admitted following surgery or only stay overnight. We studied all elective inpatient and ambulatory cases involving major therapeutic procedures performed in the state of Florida in 2018. We mapped the primary procedure to the corresponding Clinical Classification Software (CCS) category. We determined the distributions of lengths of stay overall and as stratified by CCS category, then calculated the percentage of cases that had a hospital length of stay of $\leqslant 1$ night (i.e., 0 or 1 day). A threshold of one night was selected because patients discharged home on the day of surgery have no effect on the inpatient census, and those staying overnight would either have a transient effect or no effect if observed overnight in the postoperative care unit. Among the 1,852,391 elective cases with one or more major therapeutic procedures, $65.2 \%$ (95\% lower confidence limit $[L C L]=65.1 \%)$ of cases had a length of stay of 0 days and $72.9 \%$ (95\% LCL $=72.8 \%$ ) had stay $\leqslant 1$ day. There were 38 different CCS categories for which at least $95 \%$ of patients had a length of stay of $\leqslant 1$ day. There were 28 CCS codes that identified $80 \%$ of the patients who were discharged with a length of stay $\leqslant 1$ day, showing representation of multiple surgical specialties. Our results show that even in the face of constraints imposed by a high hospital census, many categories of major therapeutic elective procedures could be performed without necessarily compromising hospital capacity. Most patients will be discharged on the day of surgery. If overnight admission is required, there would be an option to care for them in the postanesthesia care unit, thus not affecting the census. Thus, policies can reasonably be based on allowing cases with a substantial probability of at most an overnight stay rather than a blanket ban on "elective" surgery or creating a carve-out for specified surgical subspecialties. Such policies would apply to at least $72 \%$ of elective, major therapeutic surgical procedures.
\end{abstract}

Categories: Infectious Disease, Public Health

Keywords: covid-19, length of stay, patient discharge, elective surgical procedures

\section{Introduction}

The census of inpatients infected with the SARS-CoV-2 virus is high at many hospitals in the United States, interfering with the ability to take care of patients requiring treatment for conditions other than Coronavirus disease 2019 (COVID-19) [1]. Nonetheless, many patients need surgery to improve their quality of life and to prevent deterioration in health [2]. Furthermore, the curtailment of services has a substantive negative financial effect on hospitals and health systems [3].

Broad policies enacted by state or local governments to prohibit all "elective" surgical procedures to ensure sufficient hospital capacity for pandemic patients may be unnecessarily restrictive because they do not consider the lack of impact from many elective cases on the hospital census [4]. For example, there would be no rationale to restrict patients from having elective cataract surgery based solely on concern on the availability of inpatient beds because those patients are rarely admitted to hospitals following their procedure [5,6]. On the other hand, there could be a reason to restrict care based on other factors such as the absence of sufficient quantities of personal protective equipment, lack of availability of timely preoperative reverse transcriptase-polymerase chain reaction testing for SARS-CoV-2, or inadequate numbers of healthy medical personnel [7]. For example, in Florida, the executive order by Governor DeSantis that stopped all elective surgery was issued on March 20, 2020, because “... appropriate steps must be taken to conserve all medical supplies, including personal protective equipment...” [8].

We are unaware of any studies of the distributions of the length of stay for the broad range of surgical 
procedures performed at hospitals and ambulatory surgery centers [9]. However, procedures classified by the Current Procedure Terminology ${ }^{\circledR}$ (CPT) or the International Classification of Diseases, version 10, Procedure Classification System (ICD-10-PCS) codes can be combined to count statewide surgical characteristics $[10,11]$. We designed the current study to determine the distributions of the length of stay among classes of major therapeutic, surgical procedures, based on mapping the performed CPT or ICD-10-PCS codes to Clinical Classification Software (CCS) categories. The objective was to quantify the percentage of cases with major therapeutic procedures unlikely to have postoperative admission for longer than one night.

We selected a threshold of an overnight stay in the hospital for three reasons. First, for patients reliably discharged on the same day of admission (i.e., length of stay $=0$ days), considerations related to hospital admission are moot [6]. Second, ambulatory surgery centers represent a distinct class of facilities that are often geographically distant from hospitals where patients can receive care [12]. Third, for patients reliably staying overnight and discharged the next day (i.e., length of stay $=1$ day), the impact on the hospital census would be transient. If the hospital reached capacity, then the current day's elective patients would be kept in the post-anesthesia care unit (PACU) [12] and the next-day elective surgical list would be canceled. The effect on the census would be immaterial if the patients were kept in the PACU overnight and then discharged home the next day. Nelson et al. demonstrated that patients with an expected length of stay $\leqslant 1$ day who were held in the PACU overnight had a smaller difference between their expected and actual lengths of stay compared to patients not staying in the PACU overnight ( -0.096 days, $95 \%$ CI -0.30 to -0.031 days) [13].

The primary goal of this study was to provide the post-procedure length of stay data to public health policymakers at the governmental level to better inform their decisions with respect to allowing patients to receive elective surgical care during the COVID-19 pandemic.

\section{Materials And Methods}

The University of Miami Institutional Review Board determined on July 13, 2020, that this research does not meet the regulatory definition of human subjects research.

\section{Data sources}

We obtained from Florida Health publicly available data for inpatient hospitalizations and ambulatory surgical procedures between January 1, 2018, and December 31, 2018 [14] subject to a data use agreement dated May 28, 2019. These data included every surgical case at every non-federal hospital in Florida [15]. To identify whether an ICD-10-PCS code was for a major therapeutic procedure (i.e., procedure class $=4$ ), we used the "Procedure Classes for ICD-10-PCS" file from the Healthcare Cost and Utilization Project [16]. To determine if a CPT code was for a major therapeutic procedure, we determined if the associated surgery flag field for the code had a value of "narrow." For mapping CPT procedure codes (the taxonomy used in the ambulatory database) to the corresponding CCS category, we used the "2019 CCS-Services and Procedures Software" [17]. For mapping the ICD-10-PCS codes (the taxonomy used in the inpatient database) to the relevant CCS category, we used the "CCS for ICD-10-PCS Procedures, v2020.1" crosswalk files from the Healthcare Cost and Utilization Project [18]. To determine which of the listed ambulatory procedures was primary, we used the "April 2018 Physician Fee schedule from the Centers for Medicare and Medicaid Services" to map the CPT code to the work relative value units and percentage attributed in intraoperative care [19]. This approach for identification of the primary procedure for cases was used previously for statewide analyses of (a) surgeon cases per day on dates with at least one case and (b) growth in surgeon cases per week from one year to another $[9,10]$.

\section{Elective case inclusion criteria for major therapeutic procedures}

For each of the inpatient admissions, the ICD-10-PCS code for the performed primary procedure related to the reason for the hospitalization was identified along with the length of stay in days, the offset in days from admission to when the procedure was performed, emergency room charges, the admission priority of the admission, and the location source of the admission. Elective major therapeutic procedures were identified [20] by the ICD-10-PCS code having a procedure class $=4$, performed on the day of admission (i.e., 0 days), having no emergency room charges for the admission, and where the admission priority was not listed as urgent or emergent. (There were 58 patients whose admission source was listed as the emergency room, no emergency room charges, and an admission priority of elective; these patients were included.) Thus, from the inpatient database, we included only non-hospitalized patients who had a scheduled major therapeutic procedure on the day of admission. For the studied 649,962 cases, the primary ICD-10-PCS code was mapped to the relevant CCS category.

For all ambulatory cases, the database included the CPT codes for all procedures performed during the encounter, but, among these, the primary procedure was not identified. We, therefore, inferred which of the major therapeutic CPT codes was primary. After eliminating all CPT codes that were not for a major therapeutic procedure, we determined the primary procedure by determining the $\mathrm{CPT}$ with the largest value of operative work (i.e., work relative value units $\times$ percent attributed to the OR). We then mapped that CPT code to the relevant CCS category. None of the cases had a tie for operative work that mapped to different 
CCS categories. We considered all 1,202,429 ambulatory cases to be elective. The ambulatory file included a disposition code indicating if the patient was transferred to a hospital rather than being discharged home or to a non-hospital location (e.g., a nursing home). Ambulatory surgery without hospital admission was counted as having a hospital length of stay of 0 days. If the patient was transferred to a hospital from the ambulatory surgery center, we inferred that the length of stay was $>1$ day.

Inpatient surgery accounted for 649,962 cases, and ambulatory surgery accounted for 1,202,429 cases.

\section{Calculations}

From the combined inpatient and ambulatory cases, we calculated the percentage of cases where the length of stay was $\leqslant 1$ day. This included patients who were discharged on the day of surgery (i.e., 0 -day length of stay) or who stayed overnight (e.g., observation status, extended recovery, planned one-day inpatient admission). For each CCS category, we calculated the percentage of cases for that CCS where the length of stay was $\leqslant 1$ day. We determined the Clopper-Pearson conservative $95 \%$ one-sided upper and lower confidence limits (UCL, LCL, respectively) for the percentage of ambulatory patients transferred to a hospital from the ambulatory surgery center and for the percentage of patients with a length of stay $\leqslant 1$ day, respectively.

Our study was a descriptive analysis of the 1,852,391 elective surgical cases during 2018 in Florida in which the primary procedure was an elective, major therapeutic procedure; thus, no power analysis was performed.

\section{Results}

Among the 1,852,391 elective cases that included a major therapeutic procedure, $65.2 \%(95 \% \mathrm{LCL}=65.1 \%)$ of cases had a length of stay of 0 days and $72.9 \%$ ( $95 \%$ LCL $=72.8 \%$ ) had stay $\leqslant 1$ day (Figure 1 ). These were slight underestimates because they excluded $0.051 \%$ of the $1,202,429$ ambulatory surgery cases where the patient was transferred, following surgery, to a hospital (95\% UCL $=0.055 \%$ ).

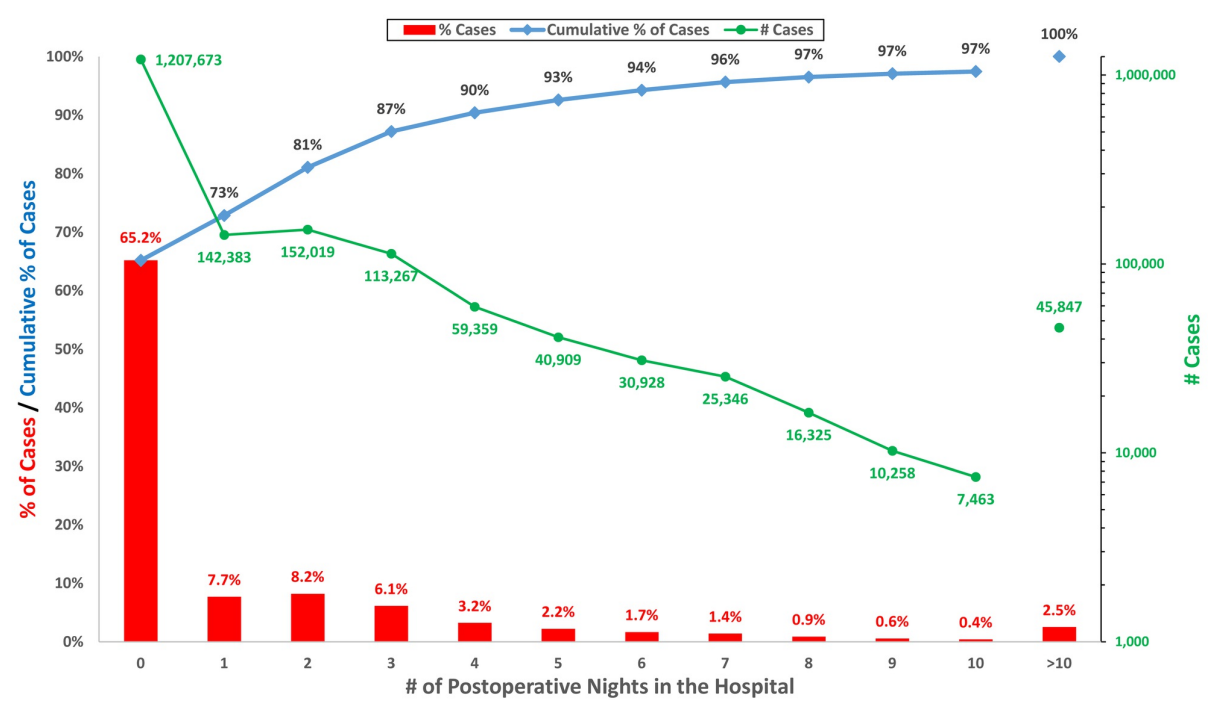

FIGURE 1: Distribution of postoperative length of stay following all elective, major therapeutic surgical procedures in the state of Florida in 2018.

The red bars indicate the percentages of the $1,852,391$ cases for each listed length of stay. The blue line represents the cumulative distribution of the lengths of stay. The green line is the count of cases for each of the listed lengths of stay. A discharge on the day of surgery corresponds to 0 nights in the hospital (length of stay $=0$ days), while an overnight stay would correspond to a one-day length of stay.

Table 1 provides a list of the 101 CCS categories for elective, major therapeutic procedures, and with at least 
100 patients having a length of stay of $\leqslant 1$ day. There were 38 different CCS categories for which at least $95 \%$ of patients had a length of stay of $\leqslant 1$ day (column 5 ). Such cases would be potentially suitable for scheduling even in the context of a hospital census constraint. There were 28 CCS codes that identified $80 \%$ of the patients who were discharged with a length of stay $\leqslant 1$ day, with multiple surgical specialties represented in this list.

\begin{tabular}{|c|c|c|c|c|c|c|}
\hline $\operatorname{ccs}$ & $\begin{array}{l}\text { Cumulative } \\
\text { proportion of } \\
\text { the } 1,350,056 \\
\text { cases with } \\
\text { length of stay } \\
\leq 1 \text { day }\end{array}$ & $\begin{array}{l}\text { Percentage } \\
\text { of the } \\
1,852,391 \\
\text { cases that } \\
\text { were of the } \\
\text { listed CCS }\end{array}$ & $\begin{array}{l}\text { Percentage of the } \\
1,852,391 \text { cases } \\
\text { that were of the } \\
\text { listed CCS and } \\
\text { with length of stay } \\
\leq 1 \text { day }\end{array}$ & $\begin{array}{l}\text { Less than } 5 \% \text { of the } \\
\text { cases in the listed } \\
\text { ccs with length of } \\
\text { stay }>1 \text { day (i.e., } \\
\text { suitable for } \\
\text { scheduling) }\end{array}$ & CCS description & $\begin{array}{l}\text { Description of the most common primary } \\
\text { procedure in the listed CCS in Florida }\end{array}$ \\
\hline 15 & $21.9 \%$ & $16.0 \%$ & $100.0 \%$ & Yes & $\begin{array}{l}\text { Lens and cataract } \\
\text { procedures }\end{array}$ & $\begin{array}{l}\text { Extracapsular cataract removal with insertion } \\
\text { of intraocular lens prosthesis, manual or } \\
\text { mechanical technique }\end{array}$ \\
\hline 160 & $28.4 \%$ & $4.9 \%$ & $95.7 \%$ & Yes & $\begin{array}{l}\text { Other therapeutic } \\
\text { procedures on muscles } \\
\text { and tendons }\end{array}$ & $\begin{array}{l}\text { Arthroscopy, shoulder, surgical; with rotator } \\
\text { cuff repair }\end{array}$ \\
\hline 162 & $32.9 \%$ & $3.9 \%$ & $82.7 \%$ & No & $\begin{array}{l}\text { Other OR therapeutic } \\
\text { procedures on joints }\end{array}$ & $\begin{array}{l}\text { Arthroscopy, shoulder, surgical; debridement, } \\
\text { extensive }\end{array}$ \\
\hline 151 & $36.3 \%$ & $2.5 \%$ & $99.9 \%$ & Yes & $\begin{array}{l}\text { Excision of semilunar } \\
\text { cartilage of the knee }\end{array}$ & $\begin{array}{l}\text { Arthroscopy, knee, surgical; with } \\
\text { meniscectomy including } \\
\text { debridement/shaving of articular cartilage }\end{array}$ \\
\hline 175 & $39.5 \%$ & $2.6 \%$ & $87.5 \%$ & No & $\begin{array}{l}\text { Other OR therapeutic } \\
\text { procedures on the skin } \\
\text { and breast }\end{array}$ & Tissue grafts, other \\
\hline 6 & $41.9 \%$ & $1.9 \%$ & $91.8 \%$ & No & $\begin{array}{l}\text { Decompression } \\
\text { peripheral nerve }\end{array}$ & $\begin{array}{l}\text { Neuroplasty and/or transposition; median } \\
\text { nerve at the carpal tunnel }\end{array}$ \\
\hline 85 & $44.3 \%$ & $1.8 \%$ & $98.5 \%$ & Yes & $\begin{array}{l}\text { Inguinal and femoral } \\
\text { hernia repair }\end{array}$ & $\begin{array}{l}\text { Laparoscopy, surgical; repair initial inguinal } \\
\text { hernia }\end{array}$ \\
\hline 124 & $46.4 \%$ & $2.9 \%$ & $55.0 \%$ & No & $\begin{array}{l}\text { Hysterectomy, } \\
\text { abdominal, and vaginal }\end{array}$ & $\begin{array}{l}\text { Laparoscopy, surgical, with total } \\
\text { hysterectomy, for uterus } 250 \mathrm{~g} \text { or less; with } \\
\text { the removal of tube(s) and/or ovary(s) }\end{array}$ \\
\hline 14 & $48.6 \%$ & $1.6 \%$ & $100.0 \%$ & Yes & Glaucoma procedures & Iridotomy/iridectomy by laser surgery \\
\hline 172 & $50.7 \%$ & $1.6 \%$ & $93.4 \%$ & No & Skin graft & $\begin{array}{l}\text { Adjacent tissue transfer or rearrangement, } \\
\text { forehead, cheeks, chin, mouth, neck, axillae, } \\
\text { genitalia, hands and/or feet; defect } 10 \mathrm{sq} \mathrm{cm} \\
\text { or less }\end{array}$ \\
\hline 84 & $52.7 \%$ & $1.6 \%$ & $92.3 \%$ & No & $\begin{array}{l}\text { Cholecystectomy and } \\
\text { common duct } \\
\text { exploration }\end{array}$ & Laparoscopy, surgical; cholecystectomy \\
\hline 142 & $54.7 \%$ & $1.7 \%$ & $85.6 \%$ & No & Partial excision bone & $\begin{array}{l}\text { Arthroscopy, shoulder, surgical; distal } \\
\text { claviculectomy including distal articular } \\
\text { surface }\end{array}$ \\
\hline 23 & $56.6 \%$ & $1.4 \%$ & $99.8 \%$ & Yes & Myringotomy & $\begin{array}{l}\text { Tympanostomy (requiring placement of } \\
\text { ventilating tube), general anesthesia }\end{array}$ \\
\hline 86 & $58.6 \%$ & $1.7 \%$ & $81.9 \%$ & No & Other hernia repair & $\begin{array}{l}\text { Repair umbilical hernia, age } 5 \text { years or older; } \\
\text { reducible }\end{array}$ \\
\hline 152 & $60.5 \%$ & $3.5 \%$ & $39.0 \%$ & No & Arthroplasty knee & $\begin{array}{l}\text { Arthroplasty, knee, condyle, and plateau; } \\
\text { medial and lateral compartments with or } \\
\text { without patella resurfacing }\end{array}$ \\
\hline 158 & $62.4 \%$ & $4.7 \%$ & $29.2 \%$ & No & Spinal fusion & $\begin{array}{l}\text { Arthrodesis, anterior interbody, including disc } \\
\text { space preparation, discectomy, } \\
\text { osteophytectomy, and decompression of }\end{array}$ \\
\hline
\end{tabular}




\section{Cureus}

\begin{tabular}{|c|c|c|c|c|c|c|}
\hline & & & & & & $\begin{array}{l}\text { spinal cord and/or nerve roots; cervical below } \\
\text { C2 }\end{array}$ \\
\hline 30 & $64.1 \%$ & $1.3 \%$ & $98.1 \%$ & Yes & $\begin{array}{l}\text { Tonsillectomy and/or } \\
\text { adenoidectomy }\end{array}$ & $\begin{array}{l}\text { Tonsillectomy and adenoidectomy; younger } \\
\text { than age } 12\end{array}$ \\
\hline 166 & $65.9 \%$ & $1.3 \%$ & $99.2 \%$ & Yes & $\begin{array}{l}\text { Lumpectomy, } \\
\text { quadrantectomy of } \\
\text { breast }\end{array}$ & Mastectomy, partial \\
\hline 161 & $67.5 \%$ & $1.3 \%$ & $87.5 \%$ & No & $\begin{array}{l}\text { Other OR therapeutic } \\
\text { procedures on bone }\end{array}$ & Removal of implant; deep \\
\hline 19 & $69.0 \%$ & $1.1 \%$ & $99.7 \%$ & Yes & $\begin{array}{l}\text { Other therapeutic } \\
\text { procedures on eyelids, } \\
\text { conjunctiva, cornea }\end{array}$ & $\begin{array}{l}\text { Excision or transposition of pterygium; with } \\
\text { graft }\end{array}$ \\
\hline 61 & $70.5 \%$ & $1.6 \%$ & $67.8 \%$ & No & $\begin{array}{l}\text { Other OR procedures } \\
\text { on vessels other than } \\
\text { head and neck }\end{array}$ & $\begin{array}{l}\text { Revascularization, endovascular, open or } \\
\text { percutaneous, femoral, popliteal artery(s), } \\
\text { unilateral; with atherectomy }\end{array}$ \\
\hline 67 & $71.9 \%$ & $1.3 \%$ & $84.6 \%$ & No & $\begin{array}{l}\text { Other therapeutic } \\
\text { procedures, hemic and } \\
\text { lymphatic system }\end{array}$ & $\begin{array}{l}\text { Biopsy or excision of lymph node(s); open, } \\
\text { deep axillary node(s) }\end{array}$ \\
\hline 48 & $73.3 \%$ & $1.0 \%$ & $91.7 \%$ & No & $\begin{array}{l}\text { Insertion, revision, } \\
\text { replacement, removal of } \\
\text { cardiac pacemaker or } \\
\text { cardioverter/defibrillator }\end{array}$ & $\begin{array}{l}\text { Insertion or replacement of permanent } \\
\text { implantable defibrillator system, with } \\
\text { transvenous lead(s), single or dual chamber }\end{array}$ \\
\hline 33 & $74.5 \%$ & $1.1 \%$ & $83.4 \%$ & No & $\begin{array}{l}\text { Other OR therapeutic } \\
\text { procedures on nose, } \\
\text { mouth and pharynx }\end{array}$ & $\begin{array}{l}\text { Submucous resection inferior turbinate, } \\
\text { partial or complete, any method }\end{array}$ \\
\hline 118 & $75.8 \%$ & $0.9 \%$ & $98.0 \%$ & Yes & $\begin{array}{l}\text { Other OR therapeutic } \\
\text { procedures, male } \\
\text { genital }\end{array}$ & $\begin{array}{l}\text { Laser vaporization of prostate, including } \\
\text { control of postoperative bleeding, complete }\end{array}$ \\
\hline 3 & $77.0 \%$ & $1.1 \%$ & $85.2 \%$ & No & $\begin{array}{l}\text { Laminectomy, excision } \\
\text { intervertebral disc }\end{array}$ & $\begin{array}{l}\text { Laminotomy (hemilaminectomy), with } \\
\text { decompression of nerve root(s), including } \\
\text { partial facetectomy, foraminotomy and/or } \\
\text { excision of herniated intervertebral disc; one } \\
\text { interspace, lumbar }\end{array}$ \\
\hline 153 & $78.2 \%$ & $2.2 \%$ & $39.8 \%$ & No & $\begin{array}{l}\text { Hip replacement, total } \\
\text { and partial }\end{array}$ & $\begin{array}{l}\text { Arthroplasty, acetabular, and proximal } \\
\text { femoral prosthetic replacement, with or } \\
\text { without autograft or allograft }\end{array}$ \\
\hline 20 & $79.3 \%$ & $0.8 \%$ & $99.7 \%$ & Yes & $\begin{array}{l}\text { Other intraocular } \\
\text { therapeutic procedures }\end{array}$ & $\begin{array}{l}\text { Vitrectomy, mechanical, pars plana approach; } \\
\text { with the removal of internal limiting } \\
\text { membrane of retina, includes, if performed, } \\
\text { intraocular tamponade }\end{array}$ \\
\hline 28 & $80.4 \%$ & $0.8 \%$ & $99.8 \%$ & Yes & $\begin{array}{l}\text { Plastic procedures on } \\
\text { nose }\end{array}$ & $\begin{array}{l}\text { Septoplasty or submucous resection, with or } \\
\text { without cartilage scoring, contouring or } \\
\text { replacement with graft }\end{array}$ \\
\hline 143 & $81.3 \%$ & $0.7 \%$ & $100.0 \%$ & Yes & $\begin{array}{l}\text { Bunionectomy or repair } \\
\text { of toe deformities }\end{array}$ & Correction, hammertoe \\
\hline 132 & $82.1 \%$ & $0.7 \%$ & $90.1 \%$ & No & $\begin{array}{l}\text { Other OR therapeutic } \\
\text { procedures, female } \\
\text { organs }\end{array}$ & Laparoscopy, surgical, colpopexy \\
\hline 147 & $82.9 \%$ & $0.7 \%$ & $81.6 \%$ & No & $\begin{array}{l}\text { Treatment, fracture, or } \\
\text { dislocation of lower } \\
\text { extremity (other than } \\
\text { hip or femur) }\end{array}$ & Open treatment of distal fibular \\
\hline 9 & $83.6 \%$ & $0.8 \%$ & $69.3 \%$ & No & $\begin{array}{l}\text { Other OR therapeutic } \\
\text { nervous system } \\
\text { procedures }\end{array}$ & $\begin{array}{l}\text { Insertion or replacement of peripheral or } \\
\text { gastric neurostimulator pulse generator or } \\
\text { receiver, direct or inductive coupling }\end{array}$ \\
\hline
\end{tabular}




\section{Cureus}

\begin{tabular}{|c|c|c|c|c|c|c|}
\hline 154 & $84.4 \%$ & $0.9 \%$ & $61.4 \%$ & No & $\begin{array}{l}\text { Arthroplasty other than } \\
\text { hip or knee }\end{array}$ & $\begin{array}{l}\text { Arthroplasty, glenohumeral joint; total } \\
\text { shoulder }\end{array}$ \\
\hline 74 & $85.1 \%$ & $1.0 \%$ & $53.3 \%$ & No & $\begin{array}{l}\text { Gastrectomy, partial } \\
\text { and total }\end{array}$ & $\begin{array}{l}\text { Gastrectomy, partial, distal; with Roux-en-Y } \\
\text { reconstruction }\end{array}$ \\
\hline 119 & $85.8 \%$ & $0.7 \%$ & $75.3 \%$ & No & $\begin{array}{l}\text { Oophorectomy, } \\
\text { unilateral and bilateral }\end{array}$ & $\begin{array}{l}\text { Laparoscopy, surgical; with the removal of } \\
\text { adnexal structures }\end{array}$ \\
\hline 148 & $86.5 \%$ & $0.6 \%$ & $81.1 \%$ & No & $\begin{array}{l}\text { Other fracture and } \\
\text { dislocation procedure }\end{array}$ & $\begin{array}{l}\text { Open treatment of metacarpal fracture, } \\
\text { single, includes internal fixation, when } \\
\text { performed, each bone }\end{array}$ \\
\hline 113 & $87.1 \%$ & $0.5 \%$ & $92.7 \%$ & No & $\begin{array}{l}\text { Transurethral resection } \\
\text { of prostate }\end{array}$ & $\begin{array}{l}\text { Transurethral electrosurgical resection of } \\
\text { prostate, including control of postoperative } \\
\text { bleeding, complete }\end{array}$ \\
\hline 57 & $87.7 \%$ & $0.5 \%$ & $99.9 \%$ & Yes & $\begin{array}{l}\text { Creation, revision, and } \\
\text { removal of } \\
\text { arteriovenous fistula or } \\
\text { vessel-to-vessel } \\
\text { cannula for dialysis }\end{array}$ & $\begin{array}{l}\text { Arteriovenous anastomosis, open; direct, any } \\
\text { site }\end{array}$ \\
\hline 51 & $88.4 \%$ & $0.7 \%$ & $68.6 \%$ & No & $\begin{array}{l}\text { Endarterectomy, vessel } \\
\text { of head and neck }\end{array}$ & $\begin{array}{l}\text { Thromboendarterectomy, including patch } \\
\text { graft, if performed; carotid, vertebral, } \\
\text { subclavian, by neck incision }\end{array}$ \\
\hline 10 & $89.0 \%$ & $0.5 \%$ & $90.0 \%$ & No & $\begin{array}{l}\text { Thyroidectomy, partial } \\
\text { or complete }\end{array}$ & Thyroidectomy, total or complete \\
\hline 106 & $89.6 \%$ & $0.4 \%$ & $99.9 \%$ & Yes & $\begin{array}{l}\text { Genitourinary } \\
\text { incontinence } \\
\text { procedures }\end{array}$ & Sling operation for stress incontinence \\
\hline 129 & $90.1 \%$ & $0.4 \%$ & $99.6 \%$ & Yes & $\begin{array}{l}\text { Repair of cystocele and } \\
\text { rectocele, obliteration } \\
\text { of vaginal vault }\end{array}$ & Combined anteroposterior colporrhaphy \\
\hline 12 & $90.6 \%$ & $0.5 \%$ & $79.5 \%$ & No & $\begin{array}{l}\text { Other therapeutic } \\
\text { endocrine procedures }\end{array}$ & $\begin{array}{l}\text { Parathyroidectomy or exploration of } \\
\text { parathyroid(s) }\end{array}$ \\
\hline 169 & $91.1 \%$ & $0.4 \%$ & $100.0 \%$ & Yes & $\begin{array}{l}\text { Debridement of wound, } \\
\text { infection, or burn }\end{array}$ & $\begin{array}{l}\text { Debridement, muscle, and/or fascia; first } 20 \\
\text { sq cm or less }\end{array}$ \\
\hline 145 & $91.6 \%$ & $0.4 \%$ & $98.3 \%$ & Yes & $\begin{array}{l}\text { Treatment, fracture, or } \\
\text { dislocation of radius } \\
\text { and ulna }\end{array}$ & $\begin{array}{l}\text { Open treatment of distal radial extra-articular } \\
\text { fracture or epiphyseal separation, with } \\
\text { internal fixation }\end{array}$ \\
\hline 167 & $92.0 \%$ & $0.4 \%$ & $83.4 \%$ & No & Mastectomy & Mastectomy, simple, complete \\
\hline 114 & $92.5 \%$ & $0.5 \%$ & $61.4 \%$ & No & Open prostatectomy & Cryosurgical ablation of the prostate \\
\hline 21 & $92.9 \%$ & $0.3 \%$ & $99.5 \%$ & Yes & $\begin{array}{l}\text { Other extraocular } \\
\text { muscle and orbit } \\
\text { therapeutic procedures }\end{array}$ & $\begin{array}{l}\text { Strabismus surgery, recession, or resection } \\
\text { procedure; } 1 \text { horizontal muscle }\end{array}$ \\
\hline 121 & $93.3 \%$ & $0.3 \%$ & $98.9 \%$ & Yes & $\begin{array}{l}\text { Ligation of fallopian } \\
\text { tubes }\end{array}$ & $\begin{array}{l}\text { Laparoscopy, surgical; with fulguration of } \\
\text { oviducts }\end{array}$ \\
\hline 80 & $93.6 \%$ & $0.3 \%$ & $92.2 \%$ & No & Appendectomy & Laparoscopy, surgical, appendectomy \\
\hline 96 & $94.0 \%$ & $1.1 \%$ & $22.5 \%$ & No & $\begin{array}{l}\text { Other OR lower GI } \\
\text { therapeutic procedures }\end{array}$ & Placement of seton \\
\hline 150 & $94.3 \%$ & $0.3 \%$ & $96.6 \%$ & Yes & $\begin{array}{l}\text { Division of joint } \\
\text { capsule, ligament, or } \\
\text { cartilage }\end{array}$ & $\begin{array}{l}\text { Capsulotomy; metatarsophalangeal joint, with } \\
\text { or without tenorrhaphy, each joint }\end{array}$ \\
\hline 87 & $94.7 \%$ & $0.2 \%$ & $99.9 \%$ & Yes & Laparoscopy & $\begin{array}{l}\text { Unlisted laparoscopy procedure; abdomen, } \\
\text { peritoneum, and omentum }\end{array}$ \\
\hline 144 & $95.0 \%$ & $0.3 \%$ & $82.4 \%$ & No & nt, facis & te(s), \\
\hline
\end{tabular}




\section{Cureus}

\begin{tabular}{|c|c|c|c|c|c|c|}
\hline & & & & & fracture, or dislocation & therapeutic \\
\hline 94 & $95.3 \%$ & $0.8 \%$ & $25.6 \%$ & No & $\begin{array}{l}\text { Other OR upper GI } \\
\text { therapeutic procedures }\end{array}$ & $\begin{array}{l}\text { Laparoscopy, surgical, esophagogastric } \\
\text { fundoplasty }\end{array}$ \\
\hline 59 & $95.5 \%$ & $0.3 \%$ & $62.0 \%$ & No & $\begin{array}{l}\text { Other OR procedures } \\
\text { on vessels of head and } \\
\text { neck }\end{array}$ & $\begin{array}{l}\text { Transcatheter placement of intravascular } \\
\text { stent(s), cervical carotid artery, open or } \\
\text { percutaneous, including angioplasty, when } \\
\text { performed, and radiological supervision and } \\
\text { interpretation; with distal embolic protection }\end{array}$ \\
\hline 164 & $95.8 \%$ & $0.2 \%$ & $88.0 \%$ & No & $\begin{array}{l}\text { Other OR therapeutic } \\
\text { procedures on } \\
\text { musculoskeletal system }\end{array}$ & $\begin{array}{l}\text { Graft; ear cartilage, autogenous, to nose or } \\
\text { ear }\end{array}$ \\
\hline 43 & $96.0 \%$ & $1.8 \%$ & $9.8 \%$ & No & Heart valve procedures & $\begin{array}{l}\text { Transcatheter pulmonary valve implantation, } \\
\text { percutaneous approach }\end{array}$ \\
\hline 16 & $96.3 \%$ & $0.2 \%$ & $99.7 \%$ & Yes & $\begin{array}{l}\text { Repair of retinal tear, } \\
\text { detachment }\end{array}$ & $\begin{array}{l}\text { Vitrectomy, mechanical, pars plana approach; } \\
\text { with endolaser panretinal photocoagulation }\end{array}$ \\
\hline 112 & $96.5 \%$ & $0.6 \%$ & $25.4 \%$ & No & $\begin{array}{l}\text { Other OR therapeutic } \\
\text { procedures of urinary } \\
\text { tract }\end{array}$ & $\begin{array}{l}\text { Aspiration of bladder; with insertion of } \\
\text { suprapubic catheter }\end{array}$ \\
\hline 26 & $96.6 \%$ & $0.2 \%$ & $83.5 \%$ & No & $\begin{array}{l}\text { Other therapeutic ear } \\
\text { procedures }\end{array}$ & $\begin{array}{l}\text { Cochlear device implantation, with or without } \\
\text { mastoidectomy }\end{array}$ \\
\hline 157 & $96.8 \%$ & $0.2 \%$ & $58.6 \%$ & No & $\begin{array}{l}\text { Amputation of lower } \\
\text { extremity }\end{array}$ & Amputation, toe; metatarsophalangeal joint \\
\hline 170 & $97.0 \%$ & $0.1 \%$ & $100.0 \%$ & Yes & Excision of skin lesion & Endoscopic plantar fasciotomy \\
\hline 125 & $97.1 \%$ & $0.2 \%$ & $53.4 \%$ & No & $\begin{array}{l}\text { Other excision of cervix } \\
\text { and uterus }\end{array}$ & $\begin{array}{l}\text { Laparoscopy, surgical, myomectomy, } \\
\text { excision; } 1 \text { to } 4 \text { intramural myomas with total } \\
\text { weight of } 250 \mathrm{~g} \text { or less and/or removal of } \\
\text { surface myomas }\end{array}$ \\
\hline 99 & $97.3 \%$ & $0.8 \%$ & $14.0 \%$ & No & $\begin{array}{l}\text { Other OR } \\
\text { gastrointestinal } \\
\text { therapeutic procedures }\end{array}$ & Unlisted laparoscopic procedure; liver \\
\hline 42 & $97.4 \%$ & $0.3 \%$ & $28.6 \%$ & No & $\begin{array}{l}\text { Other OR therapeutic } \\
\text { procedures on } \\
\text { respiratory system }\end{array}$ & $\begin{array}{l}\text { Laryngoscopy, direct, operative, with } \\
\text { excision of tumor and/or stripping of vocal } \\
\text { cords or epiglottis }\end{array}$ \\
\hline 22 & $97.5 \%$ & $0.1 \%$ & $99.9 \%$ & Yes & Tympanoplasty & $\begin{array}{l}\text { Tympanoplasty without mastoidectomy, } \\
\text { initial or revision; without ossicular chain } \\
\text { reconstruction }\end{array}$ \\
\hline 109 & $97.7 \%$ & $0.1 \%$ & $86.0 \%$ & No & $\begin{array}{l}\text { Procedures on the } \\
\text { urethra }\end{array}$ & Meatotomy, cutting of meatus; except infant \\
\hline 120 & $97.8 \%$ & $0.1 \%$ & $77.4 \%$ & No & $\begin{array}{l}\text { Other operations on } \\
\text { ovary }\end{array}$ & $\begin{array}{l}\text { Follicle puncture for oocyte retrieval, any } \\
\text { method }\end{array}$ \\
\hline 49 & $97.9 \%$ & $0.5 \%$ & $16.6 \%$ & No & $\begin{array}{l}\text { Other OR heart } \\
\text { procedures }\end{array}$ & $\begin{array}{l}\text { Insertion of ventricular assist device, } \\
\text { implantable intracorporeal, single ventricle }\end{array}$ \\
\hline 123 & $98.0 \%$ & $0.2 \%$ & $34.6 \%$ & No & $\begin{array}{l}\text { Other operations on } \\
\text { fallopian tubes }\end{array}$ & Tubotubal anastomosis \\
\hline 60 & $98.1 \%$ & $0.2 \%$ & $30.9 \%$ & No & $\begin{array}{l}\text { Embolectomy and } \\
\text { endarterectomy of } \\
\text { lower limbs }\end{array}$ & Excision of infected graft; extremity \\
\hline 104 & $98.2 \%$ & $0.5 \%$ & $16.6 \%$ & No & $\begin{array}{l}\text { Nephrectomy, partial, or } \\
\text { complete }\end{array}$ & Laparoscopy, surgical; partial nephrectomy \\
\hline 141 & $98.3 \%$ & $0.1 \%$ & $95.9 \%$ & Yes & $\begin{array}{l}\text { Other therapeutic } \\
\text { obstetrical procedures }\end{array}$ & Cerclage of cervix, during pregnancy; vaginal \\
\hline & & & & & & panoplasty with mastoidectomy; with \\
\hline
\end{tabular}




\section{Cureus}

\begin{tabular}{|c|c|c|c|c|c|c|}
\hline 24 & $98.4 \%$ & $0.1 \%$ & $98.6 \%$ & Yes & Mastoidectomy & $\begin{array}{l}\text { intact or reconstructed wall, without ossicular } \\
\text { chain reconstruction }\end{array}$ \\
\hline 17 & $98.5 \%$ & $0.1 \%$ & $100.0 \%$ & Yes & $\begin{array}{l}\text { Destruction of lesion of } \\
\text { retina and choroid }\end{array}$ & $\begin{array}{l}\text { Destruction of localized lesion of retina, } 1 \text { or } \\
\text { more sessions; photocoagulation }\end{array}$ \\
\hline 13 & $98.6 \%$ & $0.1 \%$ & $99.4 \%$ & Yes & Corneal transplant & Keratoplasty (corneal transplant); penetrating \\
\hline 171 & $98.7 \%$ & $0.1 \%$ & $84.3 \%$ & No & $\begin{array}{l}\text { Suture of skin and } \\
\text { subcutaneous tissue }\end{array}$ & $\begin{array}{l}\text { Secondary closure of surgical wound or } \\
\text { dehiscence, extensive or complicated }\end{array}$ \\
\hline 78 & $98.7 \%$ & $1.1 \%$ & $5.0 \%$ & No & Colorectal resection & $\begin{array}{l}\text { Excision of rectal tumor, transanal approach; } \\
\text { not including muscularis propria }\end{array}$ \\
\hline 53 & $98.8 \%$ & $0.1 \%$ & $99.9 \%$ & Yes & $\begin{array}{l}\text { Varicose vein stripping, } \\
\text { lower limb }\end{array}$ & $\begin{array}{l}\text { Stab phlebectomy of varicose veins, one } \\
\text { extremity; } 10-20 \text { stab incisions }\end{array}$ \\
\hline 174 & $98.9 \%$ & $0.1 \%$ & $99.9 \%$ & Yes & $\begin{array}{l}\text { Other non-OR } \\
\text { therapeutic procedures } \\
\text { on skin and breast }\end{array}$ & $\begin{array}{l}\text { Insertion or replacement of cranial } \\
\text { neurostimulator pulse generator or receiver, } \\
\text { direct or inductive coupling; with connection } \\
\text { to a single electrode array }\end{array}$ \\
\hline 176 & $98.9 \%$ & $0.1 \%$ & $65.5 \%$ & No & $\begin{array}{l}\text { Other organ } \\
\text { transplantation }\end{array}$ & $\begin{array}{l}\text { Ocular surface reconstruction; amniotic } \\
\text { membrane transplantation, multiple layers }\end{array}$ \\
\hline 117 & $99.0 \%$ & $0.0 \%$ & $100.0 \%$ & Yes & $\begin{array}{l}\text { Other non-OR } \\
\text { therapeutic procedures, } \\
\text { male genital }\end{array}$ & $\begin{array}{l}\text { Placement of interstitial device(s) for radiation } \\
\text { therapy guidance, prostate, single, or multiple }\end{array}$ \\
\hline 36 & $99.1 \%$ & $0.8 \%$ & $5.4 \%$ & No & $\begin{array}{l}\text { Lobectomy or } \\
\text { pneumonectomy }\end{array}$ & $\begin{array}{l}\text { Removal of lung, other than pneumonectomy; } \\
\text { single segment }\end{array}$ \\
\hline 2 & $99.1 \%$ & $0.1 \%$ & $54.6 \%$ & No & $\begin{array}{l}\text { Insertion, replacement, } \\
\text { or removal of } \\
\text { extracranial ventricular } \\
\text { shunt }\end{array}$ & $\begin{array}{l}\text { Replacement or revision of cerebrospinal } \\
\text { fluid shunt, obstructed valve, or distal } \\
\text { catheter in shunt system }\end{array}$ \\
\hline 244 & $99.2 \%$ & $0.0 \%$ & $100.0 \%$ & Yes & $\begin{array}{l}\text { Gastric bypass and } \\
\text { volume reduction }\end{array}$ & $\begin{array}{l}\text { Laparoscopy, surgical, gastric restrictive } \\
\text { procedure; removal of adjustable gastric } \\
\text { restrictive device, and subcutaneous port } \\
\text { components }\end{array}$ \\
\hline 101 & $99.2 \%$ & $0.1 \%$ & $54.5 \%$ & No & $\begin{array}{l}\text { Transurethral excision, } \\
\text { drainage, or removal } \\
\text { urinary obstruction }\end{array}$ & $\begin{array}{l}\text { Cystourethroscopy, with the removal of } \\
\text { foreign body, calculus, or ureteral stent from } \\
\text { urethra or bladder; complicated }\end{array}$ \\
\hline 55 & $99.3 \%$ & $0.3 \%$ & $14.3 \%$ & No & $\begin{array}{l}\text { Peripheral vascular } \\
\text { bypass }\end{array}$ & $\begin{array}{l}\text { Bypass graft, with other than vein; femoral- } \\
\text { femoral }\end{array}$ \\
\hline 146 & $99.3 \%$ & $0.2 \%$ & $18.6 \%$ & No & $\begin{array}{l}\text { Treatment, fracture, or } \\
\text { dislocation of hip and } \\
\text { femur }\end{array}$ & $\begin{array}{l}\text { Treatment of slipped femoral epiphysis; by } \\
\text { single or multiple pinning, in situ }\end{array}$ \\
\hline 63 & $99.4 \%$ & $0.0 \%$ & $98.4 \%$ & Yes & $\begin{array}{l}\text { Other non-OR } \\
\text { therapeutic } \\
\text { cardiovascular } \\
\text { procedures }\end{array}$ & $\begin{array}{l}\text { Insertion of intravascular vena cava filter, } \\
\text { endovascular approach including vascular } \\
\text { access, vessel selection, and radiological } \\
\text { supervision and interpretation, } \\
\text { intraprocedural roadmapping, and imaging } \\
\text { guidance, when performed }\end{array}$ \\
\hline 149 & $99.4 \%$ & $0.0 \%$ & $99.8 \%$ & Yes & Arthroscopy & $\begin{array}{l}\text { Arthroscopy, shoulder, surgical; with the } \\
\text { removal of loose body or foreign body }\end{array}$ \\
\hline 1 & $99.5 \%$ & $0.2 \%$ & $13.4 \%$ & No & $\begin{array}{l}\text { Incision and excision of } \\
\text { CNS }\end{array}$ & $\begin{array}{l}\text { Revision or removal of intracranial } \\
\text { neurostimulator electrodes }\end{array}$ \\
\hline 95 & $99.5 \%$ & $0.0 \%$ & $100.0 \%$ & Yes & $\begin{array}{l}\text { Other non-OR lower Gl } \\
\text { therapeutic procedures }\end{array}$ & Destruction of lesion(s), anus, extensive \\
\hline 159 & $99.5 \%$ & $0.0 \%$ & $99.8 \%$ & Yes & $\begin{array}{l}\text { Other diagnostic } \\
\text { procedures on } \\
\text { musculoskeletal system }\end{array}$ & $\begin{array}{l}\text { Arthrotomy, radiocarpal or midcarpal joint, } \\
\text { with exploration, drainage, or removal of } \\
\text { foreign body }\end{array}$ \\
\hline
\end{tabular}




\section{Cureus}

\begin{tabular}{|c|c|c|c|c|c|c|}
\hline 134 & $99.6 \%$ & $2.5 \%$ & $0.9 \%$ & No & Cesarean section & Cesarean delivery only; \\
\hline 29 & $99.6 \%$ & $0.0 \%$ & $99.8 \%$ & Yes & Oral and dental Services & Alveoloplasty, each quadrant \\
\hline 103 & $99.6 \%$ & $0.1 \%$ & $27.7 \%$ & No & $\begin{array}{l}\text { Nephrotomy and } \\
\text { nephrostomy }\end{array}$ & Nephrolithotomy; removal of calculus \\
\hline 90 & $99.7 \%$ & $0.2 \%$ & $10.8 \%$ & No & $\begin{array}{l}\text { Excision, lysis } \\
\text { peritoneal adhesions }\end{array}$ & Enterolysis (freeing of intestinal adhesion) \\
\hline 168 & $99.7 \%$ & $0.0 \%$ & $51.1 \%$ & No & $\begin{array}{l}\text { Incision and drainage, } \\
\text { skin and subcutaneous } \\
\text { tissue }\end{array}$ & $\begin{array}{l}\text { Incision and drainage, deep abscess or } \\
\text { hematoma, soft tissues of neck or thorax; }\end{array}$ \\
\hline 56 & $99.7 \%$ & $0.1 \%$ & $14.4 \%$ & No & $\begin{array}{l}\text { Other vascular bypass } \\
\text { and shunt, not heart }\end{array}$ & Venous anastomosis, open; portocaval \\
\hline
\end{tabular}

TABLE 1: Proportions of cases with a length of stay of 0 or 1 day among all $n=1,845,011$ elective major therapeutic cases performed in Florida in 2018, stratified by CCS category.

CCS, Clinical Classifications software; OR, operating room, CNS: central nervous system, Gl: gastrointestinal.

\section{Discussion}

Our results show that even in the face of constraints imposed by a high hospital census, such as for many hospitals currently dealing with an influx of COVID-19 inpatients, many categories of major therapeutic elective procedures could be performed without compromising hospital capacity. For most of these cases (65\%), patients would be sent home on the same day of admission, making consideration of hospital census irrelevant. Even when an overnight stay is needed, ambulatory patients could be recovered in the PACU, again not affecting the inpatient census [1]. We identified many individual CCS categories, comprising multiple surgical specialties, in which the likelihood of hospital admission for more than an overnight stay is less than 5\%. Thus, statewide or provincial policies should not create a carve-out policy for elective surgery such as "ophthalmology only." Rather, policies can reasonably be based on the cases with a substantial probability that the patient would have at most an overnight stay [6]. Our results show that our results apply to at least $72.8 \%$ of cases.

Previously, we studied how to combine procedures done in the inpatient and outpatient setting for purposes of counting cases by surgeon $[9,10]$ and in the current study to map to their common CCS category. Thus, there was independence regarding whether the same procedure was scheduled to be done in an ambulatory surgery center or in a hospital, with an expected discharge on the day of surgery or the next day. For policymakers and hospital administrators, the implication of our study is that when considering the limitation of elective surgical procedures in the face of the current COVID-19 pandemic, the driving force should not be to forbid "elective" cases or only to allow cases for specific specialties. Rather, they should consider the potential risk of patients requiring postoperative admission for more than one night [6]. Such flexibility might require hospitals to change their policies regarding the overnight boarding of patients in the PACU. Still, as Nelson et al. demonstrated, there was a reduction in the length of stay when patients with an expected length of stay $\leqslant 1$ day were kept in the PACU overnight [13]. We think the likely reason for this finding was that there was a motivation to discharge patients early the next morning from the PACU to make room for the next day's surgical schedule. We previously demonstrated, also using administrative data from Florida, that early morning hospital discharges of inpatients from acute care hospitals are uncommon, occurring in only 13.0\% (0.28\% standard error) of cases and unchanged between 2010 and 2018 [21].

For minor diagnostic and minor therapeutic procedures, the frequency of hospital admission is so small that there would be no reason to limit surgeries based on hospital census considerations. We were not able to analyze major diagnostic procedures because it was not possible to unambiguously determine from the database if the case utilized operating room services, including care by anesthesiology practitioners. The presence of anesthesia charges does not reliably identify who received anesthesia services. For example, anesthesia charges would be listed if a proceduralist administered a local anesthetic.

We emphasize that consideration of hospital census and the expected length of stay are not the only limitations that need to be considered when deciding if elective major therapeutic surgery can proceed during the current COVID-19 pandemic. Personal protective equipment is needed to take care of these patients, and if those supplies are in very short supply, it might not be possible to run a full elective surgical schedule [7]. If the perioperative staff pool is depleted either through contracting COVID-19, being under quarantine, or diverted to take care of inpatients, the elective surgical schedule might need to be reduced $[6,7]$. Finally, if a patient has serious comorbidities or experienced previous complications (e.g., severe postoperative vomiting) likely to result in postoperative hospitalization, he or she may not be a candidate 
for an elective procedure in the context of census constraints created by the pandemic.

Our study's public policy implications extend beyond that related to the COVID-19 pandemic, with applicability to other public health crises in which inpatient hospital resources may be strained. For example, our results can be useful in the event of an especially virulent influenza season or another viral pandemic distinct from that caused by SARS-CoV-2.

\section{Limitations}

First, the data used in this study were from a single state, so the generalizability of the findings to other states in the United States might be limited. However, Florida is a large state with a diverse population and offering a full complement of surgical services. In 2006, ambulatory surgery procedures in the USA were $62 \%$ of the ambulatory plus inpatient total [9]. The fact that in 2018 the percentage of ambulatory surgery in Florida would be greater (65\%), but not dramatically different, is reasonable. Thus, we think that the findings are likely applicable to other regions. Second, there is no mapping of patients between the ambulatory and inpatient cases, so it was not possible to determine the lengths of stay of patients who were transferred from an ambulatory surgery center to a hospital, presumably due to a complication. However, the percentage of such patients was extremely low (approximately $0.05 \%$ ), and we deliberately biased the study in counting such patients as having a length of stay of $>1$ day. Thus, the absence of the actual length of stay could not have substantively altered our results. Finally, the data available did not allow an analysis of delayed hospital admission following same-day discharge because there were no patient-level identifiers to track the occurrence of such events. To the extent that delayed readmission rates are known for a given procedure, modification of the list of acceptable procedures might be necessary.

\section{Conclusions}

There are a large and diverse number of CCS categories comprising elective, major therapeutic surgical procedures that reliably will have hospital lengths of stay of $\leqslant 1$ day. Because many patients need surgery to improve their quality of life and to avoid deterioration in medical conditions, even during a global pandemic, public policy leading to an arbitrary restriction of surgery based solely on whether the procedure is "elective" is unwarranted. Rather, policymakers should consider the likely impact of performing the primary surgical procedure, based on its CCS category, on the patient requiring hospital admission and the extent to which local hospital inpatient beds are constrained. Because the COVID-19 pandemic may remain widespread in the United States for a substantial period, the indefinite postponement of elective, major therapeutic surgery is not a viable alternative. The approach we describe is one aspect of a strategy to allow the vast majority of surgical procedures to be performed while preserving an adequate amount of hospital resources to provide care for COVD-19 patients.

\section{Additional Information}

\section{Disclosures}

Human subjects: All authors have confirmed that this study did not involve human participants or tissue. Animal subjects: All authors have confirmed that this study did not involve animal subjects or tissue. Conflicts of interest: In compliance with the ICMJE uniform disclosure form, all authors declare the following: Payment/services info: All authors have declared that no financial support was received from any organization for the submitted work. Financial relationships: All authors have declared that they have no financial relationships at present or within the previous three years with any organizations that might have an interest in the submitted work. Other relationships: All authors have declared that there are no other relationships or activities that could appear to have influenced the submitted work.

\section{References}

1. Hospital capacity and operations in the coronavirus disease 2019 (COVID-19) pandemic - planning for the nth patient. (2020). Accessed: August 13, 2020: http://https://jamanetwork.com/channels/healthforum/fullarticle/2763353?resultC.

2. Elective doesn't mean non-essential. Skip sweeping coronavirus bans, let doctors decide . (2020). Accessed: July 13, 2020: https://www.usatoday.com/story/opinion/2020/05/07/coronavirus-elective-surgery-bans-letdoctors-decide-column/5177385....

3. American Hospital Association: Hospitals and health systems face unprecedented financial pressure due to COVID-19. (2020). Accessed: July 13, 2020: https://www.aha.org/system/files/media/file/2020/05/ahacovid19-financial-impact-0520-FINAL.pdf.

4. Texas bans elective surgeries in more than 100 counties as coronavirus hospitalizations keep climbing . (2020). Accessed: July 13, 2020: https://www.texastribune.org/2020/07/09/texas-coronavirushospitalizations-elective-surgeries/.

5. Fortier J, Chung F, Su J: Unanticipated admission after ambulatory surgery: a prospective study . Can J Anaesth. 1998, 45:612-619. 10.1007/bf03012088

6. Dexter F, Elhakim M, Loftus RW, Seering MS, Epstein RH: Strategies for daily operating room management of ambulatory surgery centers following resolution of the acute phase of the COVID-19 pandemic. J Clin Anesth. 2020, 64:109854. 10.1016/j.jclinane.2020.109854

7. Dexter F, Parra MC, Brown JR, Loftus RW: Perioperative COVID-19 defense: an evidence-based approach 
for optimization of infection control and operating room management. Anesth Analg. 2020, 131:37-42. 10.1213/ane.0000000000004829

8. State of Florida, Office of the Governor: Executive Order Number 20-72 . (2020). Accessed: August 3, 2020 : https://floridapolitics.com/wp-content/uploads/2020/03/EO-20-72.pdf.

9. Cullen KA, Hall MJ, Golosinskiy A: Ambulatory surgery in the United States. Natl Health Stat Rep. 2006, $11: 1-25$.

10. Dexter F, Jarvie C, Epstein RH: At most hospitals in the State of Iowa, most surgeons' daily lists of elective cases include only 1 or 2 cases: individual surgeons' percentage operating room utilization is a consistently unreliable metric. J Clin Anesth. 2017, 42:88-92. 10.1016/j.jclinane.2017.08.016

11. Dexter F, Jarvie C, Epstein RH: Lack of generalizability of observational studies' findings for turnover time reduction and growth in surgery based on the State of Iowa, where from one year to the next, most growth was attributable to surgeons performing only a few cases per week. J Clin Anesth. 2018, 44:107-113. 10.1016/j.jclinane.2017.11.002

12. Elhag D, Dexter F, Elhakim M, Epstein RH: Many US hospital-affiliated freestanding ambulatory surgery centers are located on hospital campuses, relevant to interpretation of studies involving ambulatory surgery. J Clin Anesth. 2018, 49:88-89. 10.1016/j.jclinane.2018.06.021

13. Nelson SE, Sandberg WS, Clifton JC, Cui Y, Shotwell MS, Wanderer JP: Holding some postoperative patients overnight in the post-anesthesia care unit as a tactic to deal with hospital congestion is associated with increased resource length of stay [PREPRINT]. Perioper Care Oper Room Manag. 2020, 10.1016/j.pcorm.2020.100111

14. Agency for Health Care Administration: Order data/data dictionary. (2020). Accessed: July 13, 2020 : https://www.floridahealthfinder.gov/Researchers/OrderData/order-data.aspx.

15. AHCA patient data submission guide. (2018). Accessed: July 13, 2020: https://ahca.myflorida.com/SCHS/DataCollection/docs/DataGuideUpdate010818.pdf.

16. Healthcare cost and utilization project: procedure classes for ICD-10-PCS, v2020.1 (beta version) . (2020). Accessed: July 13, 2020: https://www.hcupus.ahrq.gov/toolssoftware/procedureicd10/pc_icd10pcs_2020_1.zip.

17. Healthcare cost and utilization project: clinical classifications software for services and procedures . (2020). Accessed: July 13, 2020: https://www.hcup-us.ahrq.gov/toolssoftware/ccs_svcsproc/ccscpt_downloading.jsp.

18. Healthcare cost and utilization project: clinical classifications software for services and procedures . (2020). Accessed: July 13, 2020: https://www.hcup-us.ahrq.gov/toolssoftware/ccs10/ccs_pr_icd10pcs_2020_1.zip.

19. Centers for Medicare and Medicaid Services: physician fee schedule, April 2018 release . (2018). Accessed: July 13, 2020: https://www.cms.gov/Medicare/Medicare-Fee-for-Service-Payment/PhysicianFeeSched/PFSRelative-Value-Files-Items/RVU18B.

20. Agency for Haleth Care Administration: overview of procedure classes (beta version) . (2020). Accessed: July 14, 2020: https://www.hcup-us.ahrq.gov/toolssoftware/procedureicd10/procedure_icd10.jsp.

21. Epstein RH, Dexter F, Diez, C: The distributions of weekday discharge times at acute care hospitals in the state of Florida were static from 2010 to 2018. J Med Sys. 2020, 44:47. 10.1007/s10916-019-1496-X 